\title{
RECURSOS PEDAGÓGICOS PARA DISPOSITIVOS MÓVEIS: UMA ANÁLISE COM FOCO NA MATEMÁTICA
}

\author{
Silvia Cristina Freitas Batista, PGIE/UFRGS, IF Fluminense Campus Campos-Centro \\ silviac@iff.edu.br \\ Patricia Alejandra Behar, NUTED-PGIE/UFRGS \\ patricia.behar@ufrgs.br \\ Liliana Maria Passerino, PGIE/UFRGS \\ liliana@cinted.ufrgs.br
}

\section{RESUMO}

Recursos pedagógicos para dispositivos móveis são fundamentais para o efetivo uso de m-learning (mobile learning - aprendizagem por meio de dispositivos móveis). Porém, é importante analisar se estes recursos levam em consideração, ou não, características que são diferenciais dessa área. Entende-se que reflexões nesse campo podem colaborar para o desenvolvimento de recursos mais adaptados à proposta de m-learning. De forma particular, este artigo tem por objetivo promover a análise e classificação de aplicativos e objetos de aprendizagem para dispositivos móveis, direcionados à matemática. Para promover as classificações, foram utilizadas três diferentes taxonomias para atividades em m-learning, identificadas na literatura. Em geral, observou-se que os recursos analisados ainda exploram pouco as especificidades dos dispositivos móveis.

Palavras-chave: matemática, m-learning, recursos pedagógicos, classificação de recursos

\section{EDUCATIONAL RESOURCES FOR MOBILE DEVICES: AN ANALYSIS WITH FOCUS ON MATHEMATICS}

\begin{abstract}
Educational resources for mobile devices are essential for the effective use of $\mathrm{m}$ learning (mobile learning - learning through mobile devices). However, it is important to consider whether these resources take into account or not, distinguishing features of this area. It is understood that discussions in this field can contribute to the development of resources best suited to the proposed m-learning. This paper particularly aims to promote the analysis and classification of applications and learning objects for mobile devices, specific to Mathematics. To promote the classifications, we used three different taxonomies for m-learning activities identified in the literature. We observed that the resources analyzed did not explore much of the specificities of mobile devices.
\end{abstract}

Keywords: Mathematics, m-learning, learning resources, classification of resources

\section{Introdução}

Mobile learning (m-learning) é um campo de pesquisa que busca analisar como os dispositivos móveis podem colaborar para a aprendizagem. O desenvolvimento de recursos pedagógicos ${ }^{1}$ para estes dispositivos são essenciais para a efetiva aplicação de m-learning.

Atividades nesta área, em geral, apresentam características como interatividade, mobilidade, trabalho em equipe, aprendizagens em contextos reais, entre outras. No 
entanto, nem todos os recursos desenvolvidos para dispositivos móveis consideram estas características. Como defendido por Patten et al. (2006), alguns recursos são particularmente adequados à aprendizagem com dispositivos móveis. São aqueles que não buscam reproduzir, ou mesmo ampliar, os atuais cenários de aprendizagem, mas sim criar novas oportunidades que não seriam possíveis sem a tecnologia móvel.

Nesse contexto, considera-se importante promover uma reflexão sobre recursos pedagógicos para dispositivos móveis. Embora essa reflexão seja necessária de maneira geral, este artigo focaliza uma área curricular em particular, a matemática.

Pesquisas relacionando m-learning e matemática são, ainda, muito recentes, mas alguns estudos já destacam vantagens no uso destes dispositivos para a aprendizagem nesta área (Calle e Vargas, 2008; Eduinnova, 2009; Baya'a e Daher, 2009). Tendo por foco a matemática, diversos recursos pedagógicos para dispositivos móveis estão sendo desenvolvidos. Entende-se, nesta abordagem, que análises sobre os mesmos podem contribuir para o desenvolvimento de recursos mais adaptados à proposta de m-learning.

Assim, este artigo tem por objetivo analisar e classificar alguns aplicativos e objetos de aprendizagem ${ }^{2}(\mathrm{OA})$ para dispositivos móveis, direcionados à matemática. Para tanto, são utilizadas três taxonomias para formas de uso de m-learning, identificadas na literatura (Naismith et al., 2004; Patten et al., 2006; Deegan e Rothwell, 2010). Os aplicativos analisados são compatíveis com celulares ou com outros dispositivos móveis, como iPhone ${ }^{\circledR}$, iPod ${ }^{\circledR}$ e iPad ${ }^{\circledR}$. Os OA considerados foram elaborados usando o MyMLE Moodle, um software para computador que permite desenvolver objetos de aprendizagem para celular.

Tendo em vista o objetivo proposto, são descritas, na seção 2, as classificações de formas de uso de m-learning, propostas por Naismith et al. (2004), Patten et al. (2006) e Deegan e Rothwell (2010). Na seção 3, são analisados e classificados recursos para m-learning direcionados à aprendizagem matemática, segundo as classificações apresentadas na seção 2. Finalizando, na seção 4, são apresentadas algumas considerações sobre o tema abordado.

\section{Formas de uso de m-learning: identificando categorias}

Segundo Deegan e Rothwell (2010), classificar formas de utilização de m-learning contribui para a compreensão de questões específicas, desafios e benefícios do uso de dispositivos móveis na educação. Nesta seção, são descritas três taxonomias para mlearning, identificadas na literatura (Naismith et al., 2004; Patten et al., 2006; Deegan e Rothwell 2010).

Naismith et al. (2004) identificam seis amplas categorias para atividades relacionadas à m-learning, de acordo com a teoria pedagógica adotada:

- Behaviorista: segundo esta abordagem, para facilitar a aprendizagem é preciso criar estímulos e oferecer reforços adequados. No contexto das tecnologias digitais, o recurso utilizado apresenta uma questão ou um problema (estímulo) que deverá ser solucionado pelo aluno (resposta). Testes de múltipla escolha enviados por mensagens de texto em celulares, por exemplo, pertencem a essa categoria;

- Construtivista: abordagem segundo a qual os alunos constroem ativamente novas idéias ou conceitos. Com m-learning, alunos são encorajados a construir seus conhecimentos, com apoio dos dispositivos móveis. Por exemplo, alunos podem participar de simulações, nas quais os dispositivos móveis permitem a imersão em sistemas dinâmicos em contextos realistas;

- Situada: envolvem atividades em contextos autênticos, tendo em vista a aprendizagem. Dispositivos móveis são bem adaptados a essa abordagem. Sistemas 
que fornecem informações em museus e galerias, com base na localização dos visitantes, são exemplos dessa categoria;

- Colaborativa: incluem atividades que promovem aprendizagem por meio da interação social. Dispositivos móveis oferecem diversos recursos para esse tipo de abordagem;

- Informal e para toda a vida: atividades que dão suporte à aprendizagem fora de contextos formais de aprendizagem. Aprendizagem informal pode ser intencional ou não. Os dispositivos móveis, por permitirem acesso à informação em qualquer tempo e lugar, são bem adaptados a esse tipo de abordagem;

- Suporte ao ensino e à aprendizagem: dispositivos móveis podem ser utilizados para acessar informações sobre horários, datas de provas, mudança de salas, entre outras, assim como acessar materiais de cursos e enviar trabalhos.

Patten et al. (2006) apresentam um framework para categorização de aplicativos para m-learning. Estes autores, assim como Naismith et al. (2004), levam em consideração as teorias pedagógicas subjacentes, mas, além disso, analisam a função dos aplicativos. O framework proposto considera sete categorias (administrativos, referenciais, interativos, micromundos, coleta de dados, sensíveis à localização e colaborativos) e identifica a teoria educacional mais claramente associada a cada uma delas. Há uma progressão entre as categorias identificadas, de modo que, em geral, uma categoria incorpora algumas das funcionalidades da anterior. As categorias iniciais reproduzem aplicativos disponíveis nos computadores, enquanto as posteriores contemplam atributos específicos de dispositivos móveis:

- Administrativos: aplicativos que focam em armazenamento e recuperação da informação, como agendas, calendários, lista de atividades, entre outros. Em geral, não são direcionados à construção do conhecimento e se apóiam muito pouco em teorias pedagógicas. Limitam-se, normalmente, a replicar, embora de forma útil, os aplicativos já disponíveis em plataformas tradicionais;

- Referenciais: incluem ferramentas estilo office, dicionários, tradutores, programas para leitura de e-books, entre outros. Fazem uso da mobilidade dos dispositivos móveis, permitindo acessar informações em qualquer tempo e lugar. Apesar de amplamente disponíveis, estes aplicativos não são pedagogicamente projetados e, como na categoria anterior, tendem a replicar aplicativos tradicionais. Em geral, são utilizados segundo uma abordagem instrucionista ${ }^{3}$ de aprendizagem;

- Interativos: de maneira geral, quando comparados com os aplicativos das categorias anteriores, os interativos tendem a ser um pouco mais criativos. Estes fazem uso dos recursos de entrada e saída dos dispositivos e incluem aplicativos do tipo teste de múltipla escolha, com feedback imediato. No entanto, existem aplicativos nesta categoria que são menos diretivos, explorando, algumas vezes, a interface touch screen de muitos dispositivos móveis. Assim, alguns, por serem do tipo "exercício e prática", podem ser associados a abordagens behavioristas, outros, mais interativos, podem ser relacionados a outros estilos de aprendizagens;

- Micromundos: são aplicativos que permitem que os alunos construam conhecimentos por meio da experimentação de modelos de domínio do mundo real. Participando dessa experimentação, os alunos são capazes de se envolver com o conteúdo, de forma inovadora. Aplicativos desta categoria são pedagogicamente mais consistentes do que os anteriores, embasados na teoria de aprendizagem construcionista ${ }^{4}$. Não são muitos os sistemas desse tipo desenvolvidos para dispositivos móveis, provavelmente devido a limitações computacionais; 
- Coleta de dados: estes aplicativos fazem uso da capacidade de dispositivos móveis para registro de dados e informações sobre o ambiente. Assim, constituem uma tentativa genuína de usar a tecnologia para criar experiências de aprendizagem que seriam inviáveis, ou pelo menos problemáticas, sem os dispositivos móveis. Geralmente, a tecnologia tem um papel pequeno e bem definido de coleta de dados, dentro de um projeto mais amplo de educação. Nesta categoria, três subcategorias, não excludentes, podem ser identificadas: i) aplicativos científicos: buscam incentivar o aluno a aprender mais sobre o ambiente, por meio do registro de informações relevantes sobre o mesmo. Aplicativos desse tipo seguem, em geral, um enfoque contextual ${ }^{5}$; ii) aplicativos reflexivos: muito comum na educação médica, estes aplicativos incentivam o registro de observações do contexto de trabalho. Posteriormente, estas observações podem ser usadas para reflexões sobre o que foi feito. Assim, estes aplicativos incentivam a prática pedagógica social reflexiva; iii) aplicativos multimídia: permitem capturar imagens, sons e vídeos para finalidades diversas. Aplicativos desse tipo, em geral, estão relacionados a uma abordagem construtivista.

- Sensíveis à localização: aplicativos desta categoria fazem uso de atributos específicos dos dispositivos móveis, permitindo ao aluno interagir com o meio ambiente. Estes utilizam sensores ou sistemas de localização e apresentam informações referenciais adequadas, indo, portanto, além da proposta da categoria anterior (Coleta de dados). Aplicativos deste tipo são úteis em atividades que envolvem visitações e experiências com realidade aumentada e, em geral, seguem uma abordagem contextual;

- Colaborativos: fazendo uso da mobilidade, ferramentas de comunicação e capacidades computacionais (como recursos de entrada e saída, rede wireless, entre outros), estes aplicativos incentivam a troca de conhecimentos, independente das localizações geográficas dos alunos. Atividades que envolvem, por exemplo, aplicativos do tipo "sensíveis à localização", muitas vezes são colaborativas. Esta categoria final, segundo os autores, é a que melhor faz uso das características da tecnologia móvel para apoio significativo de cenários de aprendizagem. Em geral, estão relacionadas a abordagens contextuais, colaborativas e construcionistas.

Patten et al. (2006) destacam as categorias "Coleta de dados", "Sensíveis a localização" e "Colaborativos" como, particularmente, adequadas à aprendizagem com dispositivos móveis, baseadas em teorias de aprendizagem construcionistas, contextuais e colaborativas. Estas três categorias incluem aplicativos que buscam fazer uso das novas oportunidades abertas com a tecnologia móvel.

Deegan e Rothwell (2010) estabelecem cinco categorias para o uso dos dispositivos móveis, de acordo com a função que desempenham na aprendizagem:

- Gerenciamento da aprendizagem: os dispositivos móveis podem ser utilizados para fazer matrícula em um curso, verificar notas e freqüências, enviar trabalhos, entre outros. Isso pode ocorrer até mesmo por meio de ambientes de aprendizagem;

- Suporte: dispositivos móveis podem dar suporte à aprendizagem presencial ou a distância. Existem duas vertentes para esta categoria: comunicação direta entre as pessoas envolvidas (chamada de voz, SMS, comunicação via redes sociais ou mensagens instantâneas) e levantamento de dados (votações, opiniões ou posicionamentos sobre o entendimento do conteúdo apresentado);

- Baseado no conteúdo: o conteúdo a ser disponibilizado nos dispositivos móveis deve ser diferente do que são apresentados nos computadores. Acessando um computador, em geral, o aluno está sentado em um local razoavelmente silencioso, no qual pode 
passar uma ou duas horas. Já com os dispositivos móveis é comum uma menor disponibilidade de tempo, em locais onde as condições do ambiente costumam contribuir para distrações. Além disso, ao disponibilizar um conteúdo, devem ser consideradas questões relacionadas ao tamanho da tela, resolução, capacidade de armazenamento, entre outras.

- Baseado no contexto: o uso de dispositivos móveis tendo em vista a aprendizagem baseada em contexto é o mais autêntico uso de m-learning, segundo os autores. Sensores incorporados no dispositivo móvel podem ajudar a interpretar o ambiente. Os resultados da aprendizagem e os materiais podem mudar com base no contexto ambiental. A realidade aumentada é considerada um uso baseado em contexto. Esta é uma combinação do mundo real e dados gerados por computador (realidade virtual), na qual objetos gráficos de computador são incorporados a imagens reais, em tempo real.

- Colaborativo: os dispositivos móveis podem apoiar situações de aprendizagens colaborativas, nas quais o aluno tem um papel ativo no processo, construindo seu conhecimento por meio da interação com o grupo. Os dispositivos móveis podem ser utilizados em grupo, por exemplo, para coletar dados em situações de contextos reais. Os dados recolhidos podem ser interpretados e realimentados pelo grupo, permitindo ver, imediatamente, os resultados do trabalho colaborativo.

Observa-se que, de maneira geral, as categorias propostas por Patten et al. (2006) são um pouco mais específicas do que as propostas por Naismith et al. (2004) e Deegan e Rothwell (2010). Patten et al. (2006) consideram dois critérios de análise (teoria pedagógica e função do aplicativo) enquanto Naismith et al. (2004) e Deegan e Rothwell (2010) analisam apenas uma (teoria pedagógica e função do aplicativo, respectivamente). No entanto, algumas categorias estão presentes nas três abordagens, ainda que com nomenclaturas diferentes. Por exemplo, pode-se entender a categoria "Situada" de Naismith et al. (2004) como correspondente à categoria "Sensíveis à localização" de Patten et al. (2006) e "Baseada no contexto" de Deegan e Rothwell (2010). Portanto, as classificações segundo os critérios "função do aplicativo" e "teoria pedagógica" se entrelaçam, mostrando que estes aspectos são bastante relacionados.

\section{Matemática e m-learning: análise de recursos}

Nesta seção, analisam-se alguns aplicativos e objetos de aprendizagem para matemática. Utilizando as três taxonomias apresentadas na seção 2, são identificadas as funções para a aprendizagem e a teoria pedagógica mais claramente associada aos recursos. Busca-se verificar se os mesmos contemplam características associadas à m-learning ou se apenas reproduzem recursos desenvolvidos para computador, sem explorar o potencial das tecnologias móveis.

Para facilitar a leitura, as propostas de Naismith et al. (2004), Patten et al.(2006) e Deegan e Rothwell (2010) serão designadas, nesta seção, como propostas A, B e C, respectivamente.

\subsection{Aplicativos}

O MobileMaths (Figura 1) é um aplicativo comercial, desenvolvido pela MobileScience $^{6}$, com versão de teste. Trata-se de um sistema computacional algébrico para celulares que permite traçar gráficos de funções em $2 \mathrm{D}$ e $3 \mathrm{D}$, resolver sistemas, trabalhar com matrizes, efetuar cálculos estatísticos, resolver equações polinomiais, calcular integrais, entre diversas outras ações. Suporta seis idiomas diferentes, dentre os quais, português. Possui versão para celulares com plataforma Java (J2ME) e para aparelho BlackBarry® e há, também, uma versão específica para celulares touch screen. 
O aplicativo apresenta um conjunto de ferramentas que pode ser utilizado de diferentes formas, sem ter uma proposta pedagógica claramente associada. Dessa forma, poderia ser classificado na categoria "Construtivista" da proposta A, de acordo com encaminhamento dado pelo professor. Mas, também é possível considerar que o mesmo pode colaborar em atividades profissionais ou em outras atividades que estejam além da aprendizagem formal, o que justificaria a opção pela categoria "Informal e para toda a vida" também da proposta $\mathrm{A}$. Na proposta B, entende-se que a categoria que melhor se adapta é a "Interativos". $\mathrm{Na} \mathrm{C}$, considera-se a categoria "Baseado em conteúdo" como a que mais se aproxima, embora os conteúdos não sejam disponibilizados diretamente, e sim ferramentas de cálculo. O Quadro 1 resume o exposto.

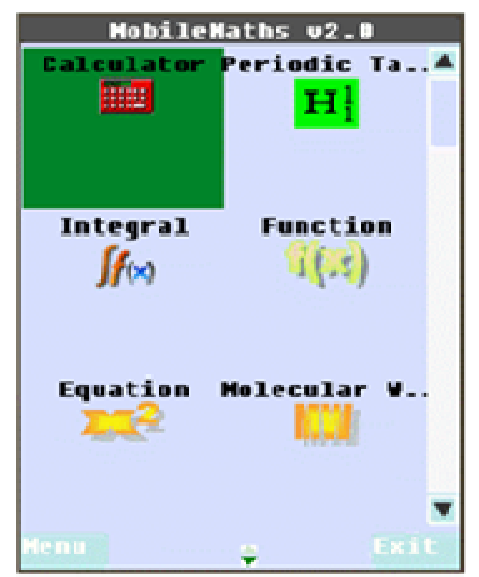

Figura 1- MobileMaths
Quadro 1 - Classificação do MobileMaths

\begin{tabular}{|l|l|}
\hline \multicolumn{1}{|c|}{ Aplicativo } & \multicolumn{1}{c|}{ MobileMaths } \\
\hline $\begin{array}{l}\text { A: Nasifmithação } \\
\text { (2004) }\end{array}$ & $\begin{array}{l}\text { Dependendo do enfoque: } \\
\text { - Construtivista } \\
\text { - Informal e para toda a vida }\end{array}$ \\
\hline $\begin{array}{l}\text { B: Patten et al. } \\
\text { (2006) }\end{array}$ & - Interativo \\
\hline $\begin{array}{l}\text { C: Deegan e } \\
\text { Rothwell (2010) }\end{array}$ & - Baseado em conteúdo \\
\hline
\end{tabular}

Em versão comercial para iPhone ${ }^{\circledR}$, iPod Touch ${ }^{\circledR}$ e iPad® há o Wolfram|Alpha App $^{7}$, desenvolvido pela empresa Wolfram, responsável pelo Mathematica ${ }^{8}$, conceituado software para computadores. O Wolfram|Alpha App inclui um sistema computacional algébrico (que apresenta passos explicativos para o alcance do resultado exibido pelo aplicativo), além de informações sobre diversas outras áreas do conhecimento, mapas e informações ambientais. Entende-se que proposta do WolframlAlpha App, devido à variedade de informações, se aproxima mais claramente da categoria "Informal e para toda a vida" da proposta A. Mas, de maneira geral, o quadro 1 poderia também resumir a análise do referido aplicativo.

O Solve2Go (Figura 2) é um aplicativo gratuito do projeto Math4Mobile ${ }^{9}$. Este permite promover comparações entre os gráficos de duas funções, selecionadas a partir de uma listagem que apresenta diversas "famílias" de funções. Os coeficientes destas funções podem ser alterados dinamicamente, o que permite a análise de diversos exemplos e favorece o estabelecimento de conjecturas. Pontos de interseção entre os gráficos podem ser identificados pelo Solve2Go, o que torna possível determinar a solução de várias equações e inequações.

Esse aplicativo apresenta uma proposta diferente dos anteriores. Possui recursos que permitem a investigação e o levantamento de hipóteses, por meio da possibilidade de execução de diversos exemplos. É possível associá-lo à categoria "Construtivista" da abordagem A, pela sua própria proposta pedagógica. Nas categorias da proposta B, a "Interativos" é que mais se aproxima, embora fique claro que essa mesma categoria está sendo utilizada para aplicativos com graus de interatividade muito distintos. Nas categorias da proposta C, a considerada mais adequada é a "Baseada em conteúdo", embora nenhum conteúdo seja explicitamente abordado e sim haja uma proposta de investigação. O quadro 2 resume as classificações. 


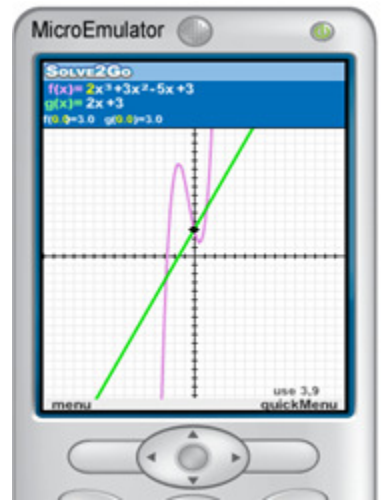

Figura 2 - Solve2Go

Quadro 2 - Classificação do Solve2Go

\begin{tabular}{|l|l|}
\hline \multicolumn{1}{|c|}{ Aplicativo } & \multicolumn{1}{|c|}{ Solve2Go } \\
Classificação & - Construtivista \\
\hline $\begin{array}{l}\text { A: Naismith et al. } \\
\text { (2004) }\end{array}$ & - Interativo \\
\hline $\begin{array}{l}\text { B: Patten et al. } \\
\text { (2006) }\end{array}$ & - Baseado em conteúdo \\
\hline $\begin{array}{l}\text { C: Deegan e } \\
\text { Rothwell (2010) }\end{array}$ & \\
\hline
\end{tabular}

O Geometry Stash $^{10}$ (Figura 3) é um aplicativo comercial, desenvolvido por Anson Liu, cujo objetivo é apresentar propriedades e teoremas de Geometria. O mesmo é compatível com iPhone ${ }^{\circ}$, iPod Touch ${ }^{\circledR}$ e iPad $®$.

Não se identificou uma categoria da proposta A que fosse adequada para o Geometry Stash. Na B, o aplicativo pode ser associado à categoria "Referenciais", uma vez o mesmo permite, basicamente, acessar informações. Na proposta C, o aplicativo é facilmente classificado como "Baseado em conteúdo" (Quadro 3).

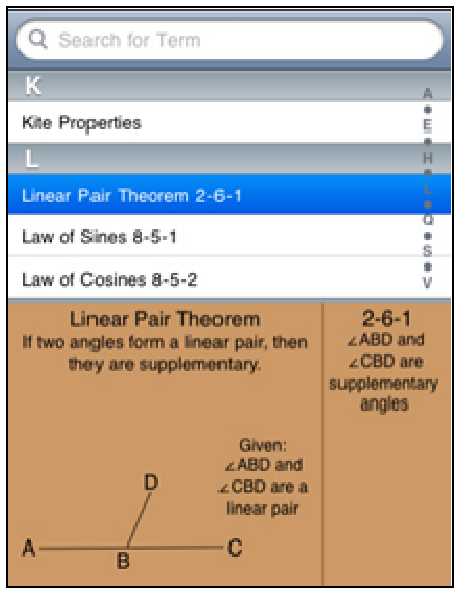

Quadro 3 - Classificação do Geometry Stash

\begin{tabular}{|l|l|}
\hline \multicolumn{1}{|c|}{ Aplicativo } & \multicolumn{1}{|c|}{ Geometry Stash } \\
\hline $\begin{array}{l}\text { A: Nassificação } \\
(\mathbf{2 0 0 4 )}\end{array}$ & ---- \\
\hline $\begin{array}{l}\text { B: Patten et al. } \\
(\mathbf{2 0 0 6})\end{array}$ & - Referencial \\
\hline $\begin{array}{l}\text { C: Deegan e } \\
\text { Rothwell }(\mathbf{2 0 1 0})\end{array}$ & - Baseado em conteúdo \\
\hline
\end{tabular}

Figura 3 - Geometry Stash

O iAptitude ${ }^{11}$ (Figura 4) é um aplicativo gratuito, desenvolvido pela empresa Softweb Solutions, compatível com iPad®. Este apresenta testes que devem ser respondidos em certo tempo, segundo três níveis de dificuldade (fácil, médio e difícil).

$\mathrm{Na}$ proposta $\mathrm{A}$, o aplicativo se adapta à categoria "Behaviorista", uma vez que são apresentados estímulos que aguardam respostas. O Quadro 4 apresenta as classificações.

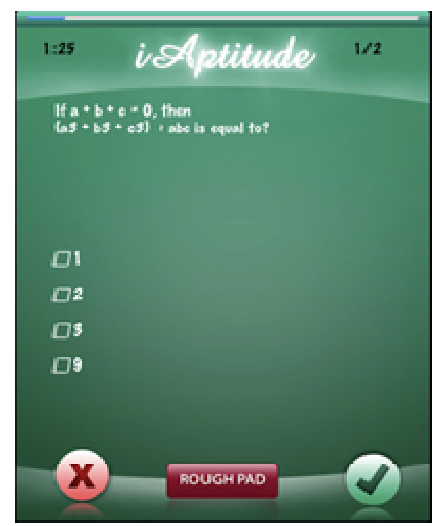

Figura 4 - iAptitude

\begin{tabular}{|l|l|}
\multicolumn{2}{|c|}{ Quadro 4-Classificação do iAptitude } \\
\hline Aplicativo & \multicolumn{1}{|c|}{ iAptitude } \\
\hline $\begin{array}{l}\text { A: Naissificação } \\
\text { (2004) }\end{array}$ & - Behaviorista et al. \\
\hline $\begin{array}{l}\text { B: Patten et al. } \\
\text { (2006) }\end{array}$ & - Interativo \\
\hline $\begin{array}{l}\text { C: Deegan e } \\
\text { Rothwell (2010) }\end{array}$ & - Baseado em conteúdo \\
\hline
\end{tabular}


Por meio do estudo realizado, foi possível observar que os aplicativos analisados são, em geral, adaptações do que já vem sendo desenvolvido para computadores e, algumas vezes, segundo abordagens pedagógicas tradicionais.

\subsection{Objetos de aprendizagem para celular: MyMLE Moodle}

O ambiente virtual de aprendizagem Moodle possui o MLE-Moodle, um plugin que permite estender suas funcionalidades para celulares. Como o próprio ambiente, o MLE-Moodle tem código-fonte livre, é gratuito e personalizável. O MLE-Moodle possui um editor (MLE-editor) para criar Mobile Learning Object (MLO), um objeto de aprendizagem próprio para o MLE-Moodle. Os MLO podem ser armazenados no celular e, posteriormente, utilizados sem requerer conexão Internet. Tais objetos podem fazer uso de todas as facilidades do MLE-Moodle, tais como: questões interativas com correção automática e possibilidade de envio de resultados ao servidor (MLE-Moodle end users, 2009).

O MLE é um recurso, em geral, utilizado em nível institucional, uma vez que requer um servidor Internet. Em versão mais simples, mais pessoal, existe o MyMLE, um programa para computador que permite criar objetos de aprendizagem para celular (com recursos semelhantes aos do MLE-editor). Depois de criados, estes objetos são selecionados e transformados em aplicativos para celular (J2ME) e podem, então, ser transferidos, juntamente com o ambiente MyMLE, por Bluetooth, por exemplo.

Os objetos apresentados na figura 5 foram elaborados com o MyMLE, no âmbito do projeto 12 “Aprendizagem com Dispositivos Móveis", como parte da pesquisa promovida pelas autoras deste artigo. Todos são questões sobre algum conteúdo matemático, o que justifica os dados do quadro 5.

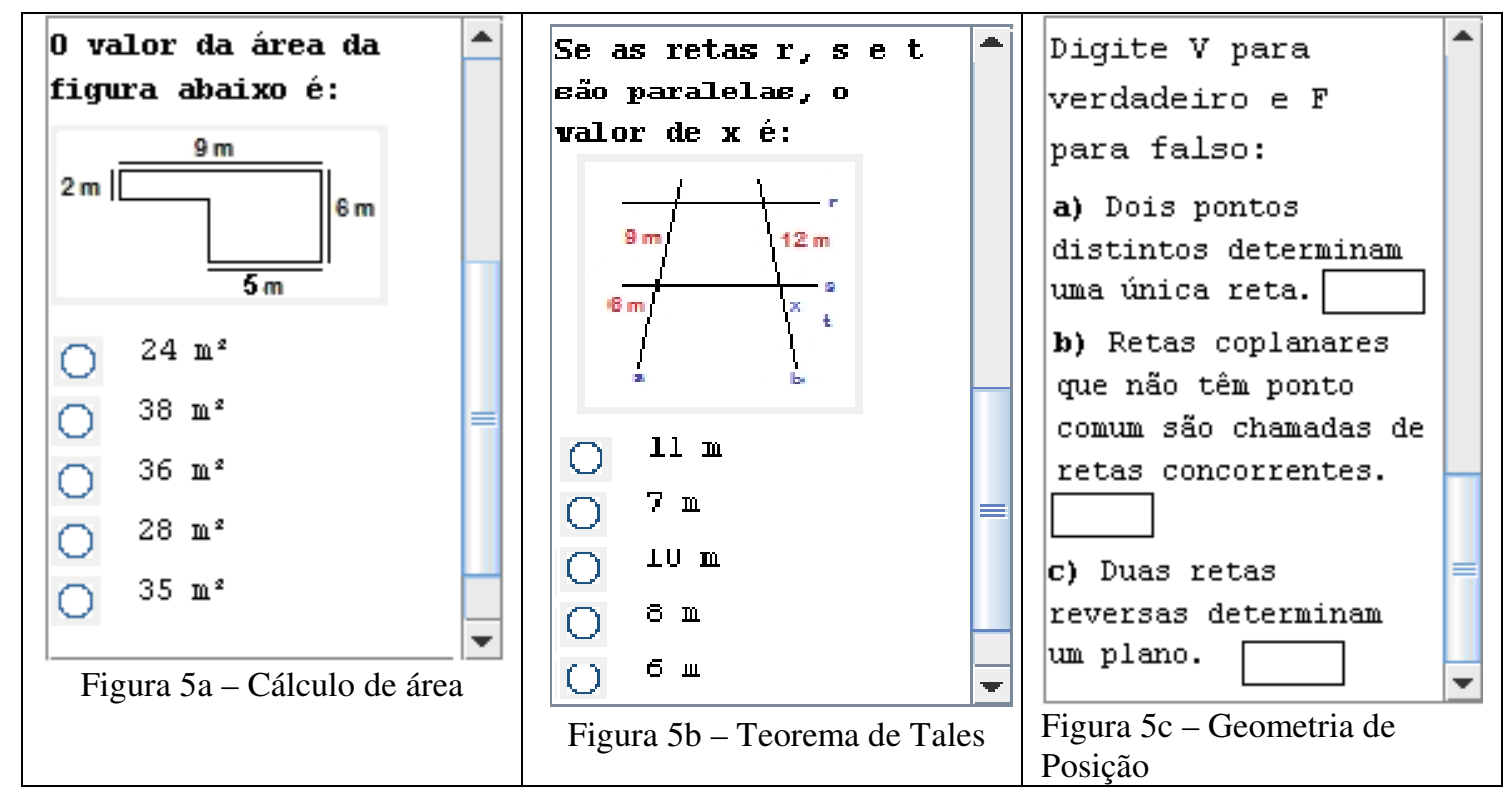

Figura 5: Objetos de aprendizagem - MyMLE

É possível elaborar objetos com mais de uma página, o que permite juntar várias questões em um único objeto ou, então, apresentar pequenos trechos teóricos e questões. Além disso, também podem ser incluídos vídeos e áudios. As ferramentas de desenvolvimento de OA do Moodle (MLE editor e o MyMLE) são, portanto, importantes contribuições para m-learning. Observa-se, no entanto, que tais ferramentas podem evoluir, de forma a possibilitar o desenvolvimento de recursos com maior grau de interatividade. Os OA apresentados na figura 5 representam pouco avanço até mesmo em relação ao material impresso tradicional. 
Quadro 5 - Classificação dos Objetos de Aprendizagem

\begin{tabular}{|l|l|}
\hline Classificação Aplicativo & \multicolumn{1}{|c|}{ Objetos de aprendizagem (Figura5) } \\
\hline A: Naismith et al. (2004) & - Behavioristas \\
\hline B: Patten et al. (2006) & - Interativos \\
\hline C: Deegan e Rothwell (2010) & - Baseados em conteúdo \\
\hline
\end{tabular}

Os recursos analisados (aplicativos e OA) podem ser acessados a qualquer tempo e lugar, por meio do dispositivo móvel correspondente. Isto favorece a mobilidade, que é uma das características de m-learning. Porém, de maneira geral, foi possível observar que os mesmos ainda refletem pouco o potencial que as tecnologias móveis possuem.

\section{Considerações finais}

A popularização dos dispositivos móveis é um aspecto positivo em termos educacionais, uma vez que favorece o alcance de um grande número de pessoas, sem requerer deslocamentos físicos. Porém, além deste aspecto, existem diversas outras características, tais como mobilidade, interatividade, aprendizagens em contextos reais, e práticas colaborativas, que têm motivado pesquisas em m-learning.

Neste artigo foram analisados aplicativos e objetos de aprendizagem para matemática em dispositivos móveis, visando verificar se os mesmos contemplavam tais características associadas à m-learning. No entanto, em geral, observou-se que os mesmos favorecem a mobilidade e a interatividade, ainda que em diferentes graus, mas exploram pouco as práticas colaborativas e a aprendizagem em contexto real.

Aplicativos com propostas mais direcionadas à investigação e à construção de conhecimentos, como o Solve2Go, representam avanços em relação a recursos tradicionais. Estes apresentam propostas que motivam o desenvolvimento do senso crítico e do raciocínio lógico. No entanto, os mesmos não deixam também de ser adaptações de softwares para computador. Aplicativos como o WolframlAlpha App, pela variedade e quantidade de informações que permitem acessar, já representam também um passo além, contribuindo para a aprendizagem informal e ao longo da vida.

Ferramentas como o MLE Editor e MyMLE, ao permitirem o desenvolvimento de recursos pedagógicos para celulares, colaboram para uma aproximação entre estes dispositivos e o contexto educacional. No entanto, em relação à matemática, observa-se que os objetos de aprendizagem obtidos são poucos dinâmicos e interativos.

Finalizando, ressalta-se que alguns recursos com propostas distintas foram associados a uma mesma categoria. Isto sinaliza que algumas categorias, como a "Interativos" de Patten et al. (2006) e a "Baseado em conteúdo" de Deegan e Rothwell (2010), podem englobar uma grande variedade de recursos, não permitindo captar certas diferenças entre os mesmos.

\footnotetext{
Notas de Texto

${ }^{1}$ Objetos e instrumentos que podem contribuir para a obtenção dos objetivos educacionais pretendidos. No contexto deste trabalho, tais objetos e instrumentos são digitais.

${ }^{2}$ Estes objetos, que visam apoiar a construção do conhecimento, podem ser criados em qualquer mídia ou formato e podem ser simples, como uma animação ou uma apresentação de slides; ou complexos, como uma simulação (Macêdo et al., 2007).

${ }^{3}$ No Instrucionismo, o aluno assume uma atitude passiva, apenas recebendo informações hierarquizadas e compartimentalizadas. Muitas vezes, mídias atuais são utilizadas segundo essa abordagem (Demo, 2006).
} 
${ }^{4}$ O Construcionismo é uma derivação do Construtivismo, estabelecida por Papert, na qual os alunos constroem ativamente seu conhecimento e sua aprendizagem por meio da construção de modelos interativos (Naismith et al., 2004).

${ }^{5}$ Entende-se a abordagem contextual como correspondente à abordagem situada, definida, brevemente, nas categorias de Naismith et al. (2004).

${ }_{7}^{6}<\mathrm{http}: / /$ www.mobile-sciences.com/>.

${ }^{7}<$ http://products.wolframalpha.com/iphone/?from=pod3>; <http://products.wolframalpha.com/ipad/index.html>.

Ressalta-se que se trata de um aplicativo comercial com preço bastante acessível.

${ }^{8}<$ http://www.wolfram.com/products/mathematica/index.html $>$.

9 <http://www.math4mobile.com/>.

$10<$ http://itunes.apple.com/pt/app/geometry-stash/id324651852?mt=8\#>. Trata-se de aplicativo comercial com preço bastante acessível.

11 <http://itunes.apple.com/pt/app/iaptitude-ipad-edition/id364607410?mt=8>.

${ }^{12} \mathrm{O}$ referido projeto é vinculado ao Instituto Federal Fluminense Campus Campos-Centro. Os objetos apresentados na figura 5 foram elaborados pelo bolsista PIBITI/CNPq Alex Botelho Mamari.

\section{Referências Bibliográficas}

BAYA'A, N.; DAHER, W. Students' perceptions of Mathematics learning using mobile phones. In: INTERNATIONAL CONFERENCE ON MOBILE AND COMPUTER AIDED LEARNING, 4., 2009, Amman, Jordan. Proceedings... Disponível em: <http://users.qsm.ac.il/cellmath/Material/Conferences/IMCL2009/students_perceptions _of_mathemat.pdf $>$ Acesso em: 28 set. 2010.

CALlE, R. C. G.; VARGAS, J. A. T. Incorporación de Tecnologías Móviles para Mejorar el Aprendizaje de Cálculo, Soportada en una Propuesta Didáctica: caso de estudio para Cálculo de Varias Variables. In: CONGRESO NACIONAL INFORMÁTICA EDUCATIVA, REDES, COMUNIDADES DE APRENDIZAJE Y TECNOLOGIA MÓVIL, 9., 2008, Barranquilla, Colombia. Actas... Barranquilla, Colombia: RIBIE, 2008. p. 1-10.

DEEGAN, R; ROTHWELL, P. A Classification of M-Learning Applications from a Usability Perspective. Journal of the Research Center for Educational Technology (RCET), v. 6, n. 1, p. 16-27, Spring 2010.

DEMO, P. Instrucionismo e Nova Mídia. In: SILVA, M. Educação Online: teorias, práticas, legislação, formação corporativa. 2. ed. São Paulo: Edições Loyola, 2006, p. 77-90.

EDUINNOVA. Tecnología Portátil en la Sala de clases: Pocket PC (Proyecto Piloto Informe $\quad$ Final). 2009. Disponível em: $<$ http://colabora.inacap.cl/sitios/ciedu/Documentos\%20compartidos/Archivos_Pag_CIE DU/Informe_final_Eduinnova.pdf>. Acesso em: 23 mar. 2010.

MACÊDO, L. N. de; CASTRO FILHO, J. A. de; MACÊDO, A. A. M.; SIQUEIRA, D. M. B.; OLIVEIRA, E. M. de; SALES, G. L.; FREIRE, R. S. Desenvolvendo O Pensamento Proporcional com o Uso de um Objeto de Aprendizagem. In: PRATA, C. L.; NASCIMENTO, A. C. A de (Org.) Objetos de aprendizagem: uma proposta de recurso pedagógico. Brasília: MEC, SEED, 2007. p.17-26.

MLE-MOODLE - END USERS. MLE-Moodle. 2009. Disponível em: $<\mathrm{http}$ ://mle.sourceforge.net/mlemoodle/index.php?lang=en >. Acesso em: 02 jun. 2010.

NAISMITH, L.; LONSDALE, P.; VAVOULA, G.; SHARPLES, M. Literature Review in Mobile Technologies and Learning. UK: NESTA Futurelab Series, 2004. PATTEN, B.; ARNEDILLO SÁNCHEZ, I.; MCGREEN, N.; CLARKE, M.; BRENNAN, E.; TANGNEY, B. Designing collaborative, constructionist and contextual applications for handheld devices. Computers \& Education, Oxford, UK: Elsevier Science Ltd, v. 46, p. 294-308, 2006. 